\title{
Treatment of Acute Diarrheal Diseases
}

\section{Sujit K Bhattacharya*}

Ultracare Hospital and Research Centre, India

*Corresponding author: Sujit Kumar Bhattacharya, MD, Ultracare Hospital and Research Centre, India, Tel: +8697462003; Email: sujitkbhattacharya@yahoo.com

\section{Mini Review}

Volume 4 Issue 2

Received Date: May 04, 2020

Published Date: May 26, 2020

DOI: $10.23880 / \mathrm{jidtm}-16000140$

\section{Abstract}

Acute diarrhoea is an important cause of morbidity and mortality in children in the developing countries. It is caused by 2025 pathogens. Dehydration is the cause of death. Treatment involves correction of dehydration, judicious use of antibiotic and feeding. Sanitation and safe water are important preventive measure. Vaccination is an attractive disease prevention strategy. Oral, effective and safe cholera vaccine has been developed.

Keywords: Diarrhoea; Treatment; Cholera; Shigellosis; Dehydration

Abbreviations: CT: Cholera Toxin; WHO: World Health Organization; ORT: Oral Rehydration Therapy; HUS: Hemolytic Uremic Syndrome.

\section{Introduction}

Acute diarrhoea is prevalent in areas where sanitation is poor and safe water is not available. Acute diarrhoea is an important cause of morbidity and mortality in children below 5 years of age. 2-3 episodes of acute diarrhoea occur annually. It is caused by 20-25 pathogens. Treatment consists of correction of dehydration and judicious use of antibiotic. In this communication, treatment of acute diarrhoea is briefly discussed, particularly cholera and shigellosis.

\section{Magnitude of the problem}

Dehydration: In acute diarrhoea, salt and water are lost from the body. In cholera, the fluid loss occurs in the small intestine due to the action of an exotoxin known as cholera toxin (CT) elaborated by the cholera Vibrio. When this fluid loss exceeds colonic absorption, diarrhoea is manifested. This results in a clinical condition known as "dehydration". Dehydration is classified into mild, moderate and severe. Mild to moderate dehydration can be treated with WHO recommended Oral Rehydration Salt Solution (Table 1). Severe dehydration requires intravenous fluid. The best intravenous fluid is Ringer's lactate followed by Normal saline.

\begin{tabular}{|c|c|c|}
\hline Ingredients & Standard ORS & Hypo-osmolar ORS \\
\hline Sodium Chloride & $3.5 \mathrm{~g}$. & $2.6 \mathrm{~g}$. \\
\hline Potassium Chloride & $1.5 \mathrm{~g}$. & $2.5 \mathrm{~g}$. \\
\hline Tri-sodium citrate dehydrate & $2.9 \mathrm{~g}$. & $13.5 \mathrm{~g}$. \\
\hline Glucose (anhydrous) & $20 \mathrm{~g}$. & 245 \\
\hline Osmolarity (mosmol/litre) & 311 & \\
\hline
\end{tabular}

Table 1: WHO recommended Oral Rehydration Salt Solution.

Cholera: Cholera is caused by Vibrio cholerae 01 and 0139. Cholera causes sporadic cases, epidemics and even pandemics [1]. The current pandemic is the $7^{\text {th }}$ pandemic which started in Celebes Island in Indonesia in 1961 and spread all over the world. Majority of cholera cases are asymptomatic. A small percentage of cases present with severe dehydration. 
In 1992, a novel strain of Vibrio designated as Vibrio cholerae 0139 Bengal appeared in Gangetic delta and caused massive epidemics in Kolkata and Dhaka and spread to many parts of the world including some developed countries.

Management of cholera is essentially correction of initial fluid deficit and maintenance due to ongoing losses. Cholera stool typically looks like Rice-water stool (flecks of mucous suspended in fluid). Antibiotic is indicated in severe cases only. Antibiotic reduces the stool volume, frequency of purging and excretion of pathogen in stool. Antibiotic of choice are many, e.g. tetracycline (500 mg 6 hourly for 3 days), Ciprofloxacin (500 mg 2 times daily for 3 days), Norfloxacin (400 mg 2 times daily for 3 days) or doxycycline( $300 \mathrm{mg}$ single doses). Oral antibiotic is preferred. Antibiotic should be given once the vomiting stops. Tetracycline resistant $V$. cholerae has been reported. Charcoal and kaolin or pectin is ineffective and not recommended. Anti-emetics cause sedation and hypotension and may interfere with Oral Rehydration therapy (ORT). Feeding during and after recovery is important and prevents malnutrition. Children should be given an extra food daily for 6 weeks.

An oral cholera vaccine [2-5] has been developed which gives protection up to $66 \%$ of the vaccines for a period of 5 years. The earlier used injectable vaccine has been discontinued because of low protection rate and reactogenecity at the side of injection.

Shigellosis: Shigellosis is manifested by passage of loose bloody stools mixed with mucus, and associated with abdominal cramps, fever, anorexia (particularly in children) and tenesmus (incomplete sense of defecation). Shigellosis is caused by Shigella spp. There are four serotypes, e.g. S. sonnei, S. boydii, S. flexneri and $S$. dysenteriae. S. sonnei and $A$. boydii cause mild dysentery and occur in developed countries. $S$. flexneri causes moderate to severe dysentery. $S$. dysenteriae type 1 causes the most severe dysentery and produce epidemics and have also caused pandemic. This stain causing dysentery is associated with fever, convulsion (children), arthritis, conjunctivitis, toxic megacolon, and Hemolytic Uremic syndrome (HUS). HUS is a triad of hemolysis, thrombocytopenia and renal failure.

Management: $S$. dysenteriae type 1 is multi-drug resistantand causes therapeutic challenge [6-8]. Currently, ciprofloxacin is the drug of choice, but even $S$. dysenteriae type 1 strains resistant to ciprofloxacin have been reported. Ceftriaxone, though given intravenously, is effective. Children should be encouraged to eat. Oral rehydration therapy is beneficial as it gives fluid and electrolytes.

Traveler's Diarrhoea: Traveler's diarrhoea is a common occurrence when travelers from developed countries travel to developing countries. The commonest cause is Enterotoxigenic Escheria Coli. Traveler's diarrhoea due to Vibrio cholerae 0139 and Shigella dysenteriae type 1 have been reported from Kolkata.

\section{References}

1. Bharati K, Bhattacharya SK (2014) Cholera outbreaks in South-East Asia. Curr Top Microbiol Immunol 379: 87116.

2. Bhattacharya SK, Sur D, Ali M, Kanungo S, You YA, et al. (2013) 5 year efficacy of a bivalent killed whole-cell oral cholera vaccine in Kolkata, India: a cluster-randomised, double-blind, placebo-controlled trial. Lancet Infect Dis 13(12): 1050-1056.

3. Roychowdhury A, Pan A, Dutta D, Mukhopadhyay AK, Ramamurthy T, et al. (2008) Emergence of tetracyclineresistant Vibrio cholerae 01 serotype Inaba, in Kolkata, India. Jpn J Infect Dis 61(2): 128-129.

4. Raychoudhuri A, Chatterjee S, Pazhani GP, Nandy RK, Bhattacharya MK, et al. (2007) Molecular characterization of recent Vibrio cholerae 01, El Tor, Inaba strains isolated from hospitalized patients in Kolkata, India. J Infect 55(5): 431-438.

5. Sur D, Dutta S, Sarkar BL, Manna B, Bhattacharya MK, et al. (2007) Occurrence, significance \& molecular epidemiology of cholera outbreaks in West Bengal. Indian J Med Res 125(6): 772-776.

6. Bhattacharya SK, Sur D, Mahalanabis D (2012) Public health significance of shigellosis. Indian Pediatr 49(4): 269-270.

7. Sur D, Ramamurthy T, Deen J, Bhattacharya SK (2004) Shigellosis: challenges \& management issues. Indian J Med Res 120(5): 454-462.

8. Niyogi SK, Sarkar K, Lalmalsawma P, Pallai N, Bhattacharya SK (2004) An outbreak of bacillary dysentery caused by quinolone-resistant Shigella dysenteriae type 1 in a northeastern state of India. J Health Popul Nutr 22(1): 97. 